\title{
Standardization of Neuroanesthesia Education: Need of the Hour and the Way Forward
}

\author{
Shobana Rajan ${ }^{1} \cdot$ Maria Bustillo ${ }^{2} \cdot$ Chanhung Lee ${ }^{3} \cdot$ W Andrew Kofke ${ }^{4}$ \\ Accepted: 1 July 2021 / Published online: 26 October 2021 \\ (c) The Author(s), under exclusive licence to Springer Science+Business Media, LLC, part of Springer Nature 2021
}

\begin{abstract}
Purpose of Review This review illustrates the evolution and progress with standardization of fellowship education in neuroanesthesiology. It provides a structured discussion around the need for curricula and framework which individual training programs in neuroanesthesiology can use to meet defined educational standards thus meeting criteria for accreditation.

Recent Findings Neuroanesthesiology training has traditionally been heterogenous around the world but international efforts from the community of neuroanesthesiology have culminated in the development of an international council for perioperative training in neuroscience in anesthesiology(ICPNT). This serves not only as an accrediting body but also creates a platform through their neuroanesthesia program relations committee for collaboration and engagement between various training programs internationally, increasing the educational standards of the individual programs and collectively increasing the overall level of standards for neuroanesthesia training. Standardized curriculum and competency-based assessments and milestones would help with narrowing the focus to quality education in neuroanesthesiology.

Summary Structured training around the three pillars of neuroanesthesiology with concomitant accreditation is expected to lead to higher education standards with better patient care. The SNACC created milestones for neuroanesthesiology training during residency and the ICPNT can now use this as a foundation for fellowship training. Having a council to accredit and standardize will likely become indispensable in creating a set path for training in neuroanesthesiology. Additionally, the flexibility built in due to the international nature would allow modified and variable pathways depending upon individual capabilities and interests. The path forward will include widespread adoption of standardization supporting the overarching goal of excellent patient outcomes around the world.
\end{abstract}

Keywords Neuroanesthesiology · Neurosurgical procedures · Neuroanesthesia education · Standardization of education · Neuroanesthesia fellowships · Accreditation of Neuroanesthesia Fellowships · Perioperative neuroscience training ·

Milestones · Competencies · Curriculum

\section{Introduction}

With increasing numbers of complex and specialized neurosurgical and neuroradiological procedures being conducted, current training and education in neuroanesthesiology should adapt to create a specialist with expertise in team based care in perioperative neurosciences providing excellent patient outcomes. As a neuroanesthesiologist and perioperative neuroscience expert, the well trained neuroanesthesiologist

This article is part of the Topical Collection on Neuroanesthesia

Shobana Rajan

shobanarajan5@gmail.com

Extended author information available on the last page of the article should be a leader capable of orchestrating the perioperative environment to optimize care and support innovations. Standardization of neuroanesthesia education and proper training will lay the foundation for acquisition of neuroanesthesiology specific clinical skills, afford a knowledge base required to optimize the central nervous system pathophysiology and support appropriate use of available peri-operative brain monitoring technologies. The challenge is to implement educational strategies that will enhance the quality of care offered in the realm of perioperative neurosciences.

This review will focus on available literature regarding:

1. Subspecialty training in anesthesiology and evolving status of neuroanesthesiology training

2. Standardized training-benefits and barriers 
3. Steps that led to promoting quality and standards in performance of neuroanesthesiology- Evolution of accreditation of neuroanesthesia fellowships

4. Adaptation of the three Pillars of neuroanesthesiology as a model to develop standardized education strategies.

5. Development of a neuroanesthesia program relations committee.

6. Curriculum and core competencies of neuroanesthesia training making standardization a practical reality.

7. Assessment and evaluation using neuroanesthesia milestones- an essential component of standardization as the future of the subspecialty evolves.

8. Conclusion

\section{Subspecialty Training in Anesthesiology and Evolving Status of Neuroanesthesiology Training}

Training during residency provides only a broad orientation to any sub-specialty but a fellowship is expected to offer skills, knowledge and experience required to be an expert in the field. There are 10 sub-specialty fellowships under the umbrella of anesthesiology accredited by ACGME: Pediatrics, Critical Care, Pain, Obstetric, Cardiac, Regional, Neurocritical care, Palliative care and hospice, Addiction Medicine and Clinical Informatics. Neuroanesthesia fellowship does not fall under this umbrella.

In recent times the growing demand of transformational innovations and exponential growth in neurosurgery, neurology and interventional neuroradiology, has created the need to have a dedicated international group of experts with the appropriate clinical, research, and educational foundation in perioperative neuroscience to improve patient outcomes and health care delivery [1]. Hence, standardization and accreditation could be the way forward for the success in future training in neuroanesthesiology.

Currently neuroanesthesia fellowship training has been heterogenous with lack of uniformity around the world in terms of both the scope of curricula offered and duration of fellowship from 6 months to three years [2, 3]. While some fellowships are independent and can be done any time after residency, some are enfolded into the residency which means the trainee does the specialized training during the time of residency. These international differences in neuroanesthesia training culture arise due to different types of funding available to support the fellowship; while in some departments, fellows are funded by the GME (Graduate Medical Education) program of the hospital, in other departments they function as junior faculty eliminating the need for dependence on government funding. To address this heterogeneity, the fellowship structure, content, duration and accreditation has been a topic of extensive discussion over the last few decades.

In cooperation with SNACC leadership, a web-based survey was distributed to all SNACC members practicing in the US ( $n=339)$ through electronic mail [4]. The survey assessed the degree of support for the accreditation of neuroanesthesia fellowship training programs on a scale of 1 (no support) to 10 (complete support), the optimal duration of a fellowship, with the choices of 6 months, 1 year, and 2 years and the importance of various elements for a neuroanesthesia fellowship curriculum on a scale of 1 (no importance) to 10 (absolute importance). Eighty percent of respondents identified a 1-year duration as the optimal training period for a neuroanesthesia fellowship. There was moderate support for accreditation.

The fellowship structure, content and accreditation will be discussed in the following sections of the manuscript.

\section{Standardized Training-Benefits And Barriers}

Any fellow who undergoes training in a standardized program would graduate with clinical skills and capabilities which are equivalent to other fellows from other standardized programs. This would allow dependability of clinical performance from fellows trained from these programs. It would entail having common guidelines for curricula, similar principles of feedback and evaluation, an accreditation body which is part of the sub-specialty society to oversee the implementation of the above. Standardization may offer many rewards such as fostering continuous quality improvement, patient safety and enable the spread of best practices. Standardization can deliver quality medical education that is globally accepted and locally active [5].

There could be a number of barriers to standardization. Standardizing curriculum may compel students to take a specific path of training by enforcing a one-size-fits all curriculum; This could limit opportunities for trainees who may want to pursue a specific skill or focused area of clinical neuroanesthesiology or research. Standard feedback and evaluations may again not do justice to a trainee seeking to excel in specific areas of interest. The way ICPNT has structured its curriculum to give flexibility to individual programs and fellows and offer global collaboration could overcome many of these barriers. Giving some flexibility to personalize the trainees experience, affording myriads of learning opportunities would certainly help achieve the ultimate expectation of achieving mastery in the field of neuroanesthesiology. 


\section{Steps that Led to Promoting Quality and Standards in Performance of Neuroanesthesiology-Evolution of Accreditation of Neuroanesthesia Fellowships}

Neuroanesthesia fellowships are a topic of unremitting interest since the early years of the Society of Neuroscience in anesthesia and critical care(SNACC) $[6,7]$.

\section{History that Led to the Foundational Concepts of Accredita-} tion The discussion and debate about fellowships ensued over the almost 50-year life of SNACC. Dr. Maurice Albin, a SNACC founder, wrote a seminal article on the need for neuroanesthesia fellowships in the fall 2008 SNACC newsletter which brought to attention many of the questions regarding fellowship accreditation [8]. Albin emphasized the remarkable changes that have transformed neuroanesthesia practices since his personal full-time involvement in 1962 and since the 1973 organization of the predecessor to SNACC-the Society of Neurosurgical Anesthesiology (SNA). This progress has been manifested by:

- the enhanced knowledge of the dynamics of brain and spinal cord physiopathology and neurochemistry and its interaction with the anesthetic state.

- the formation of neuroanesthesia fellowship Programs in many Centers.

- developing standards of care for many neuroanesthesia procedures.

- bringing neuroanesthesiology considerations into the residency and medical school curriculum.

- the formation of a well-recognized, international subspecialty society of neuroanesthesia, SNACC and other neuroanesthesia societies across the world, all with significant membership and well-defined missions and programs

- Basic laboratory and clinical research in perioperative neuroscience [9]

- the existence of the dedicated Journal of Neurosurgical Anesthesiology with Dr. James E Cottrell and Dr. John Hartung as founding Editors - now edited by Dr. Martin Smith, making this publication a focal point for world neuroanesthesiology. Additionally, the evolution of neuroanesthesia as a specialty is also evidenced by dedicated neuroanesthesia sections in other anesthesiology journals with expert neuroanesthesiologists as section editors.

Albin further made a plea for strengthening neuroanesthesiology fellowship programs and advocated that we aim for subspecialty program accreditation and eventual individual certification. Numerous articles have been published in the SNACC newsletter encouraging development of a formal fellowship curriculum. This debate resulted in peer-reviewed research indicating support for fellowships and development of curricular guidelines $[5,10]$ which have been largely incorporated into the accreditation criteria promulgated by the International Council for Perioperative Neuroscience Training (ICPNT).

The process for international accreditation of neuroanesthesia fellowships has arisen in a context of varying approaches to certification and accreditation in many nations with some controversy. Accreditation refers to an acknowledgement that a specific fellowship program provides a suitable experience to result in competent graduates in a medical specialty. Graduation from an accredited program is typically required to be eligible for certification. The American College of Graduate Medical Education (ACGME) is the most well-known and accepted organization for conferring accreditation to medical programs. Due to administrative aspects, alternate pathways to accreditation have come into place in the US such as UCNS (United Council for Neurologic Subspecialties)and CAST for neurocritical care and ICPNT for neuroanesthesiology.

International Efforts to Develop Accreditation and Standardization Concurrent with the above-noted US-based SNACC discussions, colleagues from numerous sites in the world including Europe, Canada, China, South America Mexico, Thailand, Myanmar India, and UK have been conducting parallel discussions [3, 11-18]. Sewell et al. [13] and Valero et al. [14] surveyed Canadian and European programs respectively finding a lack of uniformity in training standards and in Europe Valero noted general support to develop a neuroanesthesia fellowship accreditation system. Colleagues in a number of countries were working together through a number of organizations, including the European Society of Anesthesiology (ESA), EuroNeuro, and the European Neuroanaesthesthesia and Critical Care Interest Group (ENIG) to improve education, training, research, and guideline production.

Role of International Collaboration in the Success of Standardization of Training and Accreditation All of the already established accreditation bodies considered had a USAbased system; however, SNACC has always intended to be an international scientific society with a history of leadership by officers based in non-US nations, or who are immigrants from elsewhere (Canada, Italy, Germany, Netherlands, UK, India, Thailand, Iran). There are also several very prominent non-USA international neuroanesthesiology fellowship programs with years of experience and significant research productivity, and not including them in the vision in establishing the accreditation system was not in line with SNACC 
goals. These fellowship programs, their directors and faculty are an important resource for networking and enriching perioperative neuroscience for all trainers and trainees. Undeterred by the UCNS events and in consideration of its international scope, SNACC then considered several options and finally adopted a SNACC-based international neuroanesthesiology fellowship accreditation service.

The Novel ICPNT Was Established The novel ICPNT blueprint described a council for establishing international standards of post specialty training in the field of perioperative neuroscience that would reside administratively in SNACC. The SNACC Executive Council amended the SNACC bylaws to allow the incorporation of the council. A writing committee* was created which was tasked with creating the foundational documents for this organization, subsequently named the International Council for Perioperative Neuroscience Training (ICPNT). This name indicates that the scope of the program is international and includes all perioperative neuroscience not limiting it to Neuroanesthesiology, allowing for possible future fellowship support for neuromonitoring, neurocritical care, or other related disciplines. The writing committee, with the administrative assistance of "Ruggles Service Corporation"( https://www. rugglesamc.com/our-team/), created a web page, a charter for ICPNT and the program requirements for accreditation.

\section{International Spectrum and Scope Are Taken Into Account} Right from ICPNT Conception Notably, the program requirements are written in a way which supports international differences in regional training culture and regulations, while describing a required minimum of curriculum and clinical experience to comprise an accredited neuroanesthesiology fellowship. Moreover, the program requirements allow for different methods of funding and can be from institutional training funds or requiring fellows function as part time billing faculty. While there is educational value to any experience which includes being part time billing faculty, this is not an element of the ICPNT fellowship training paradigm. Rather it is primarily a mechanism to financially support an individual's educational experience. In addition, the concept of an enfolded fellowship during residency is also supported. This model would give consideration for the different types of training that exists globally to come under a common umbrella. The ICPNT also developed an accreditation application and an innovative process for evaluation of programs. Overall, this has resulted in increasing interest in accreditation around the world (Table 1).

System of Accreditation Is Novel Each application has two stages; the first is a formative submission by which reviewers provide feedback to the program, followed by a summative evaluation by which final accreditation is provided.
Table 1 Representative activities of the Neuro-anesthesia Program Relations Committee

Neurological manifestations of Covid-19 webinar

Survey to determine the impact of Covid-19 on fellowship training and education [17]

ICPNT journal club webinar

ICPNT case based reasoning webinar

Perfecting peer review- a master class webinar

Evaluation strategies for neuro-anesthesia fellows- A milestones approach- workshop webinar

Survey to Program Directors regarding needs assessment

Pearls in neuromonitoring webinar

Basic Conundrums in Neuroscience

Consequently, every accreditation application is associated with constructive feedback to enable improvement of the applying program. This approach reinforces the overarching goal of ICPNT to be a vehicle to standardize and improve neuroanesthesia training and care everywhere.

ICPNT Supports a Collaborative Framework of International Fellowship Programs ICPNT is to be differentiated from other accreditation systems in that.

1. It supports an international network of interactive accredited neuroanesthesiology fellowship programs (Fig. 1 Collaborative framework of accredited programs).

2. While other accreditation organizations focus solely on evaluation of programs, ICPNT provides an infrastructure for inter-program education and quality improvement. This enhances education and may provide an infrastructure for an international clinical and research network.

\section{Adaptation of the Three Pillars of Neuroanesthesiology as a Model to Develop Standardized Education Strategies}

With progress being made for the standardization of education in neuroanesthesiology, the notion of the "Three pillars of neuroanesthesiology" was suggested by the Past President of the SNACC Dr.George Mashour [19, 20]. He proposed that while neuroanesthesiologists care for neurosurgical and neurocritical care patients, it is critical to expand focus to understand and advance the foundational neuroscience of anesthesiology as well as serve as consultants regarding neurologic outcomes of surgery. He regarded this as vital to the specialty I the years to come.

The three pillars are as follows: 


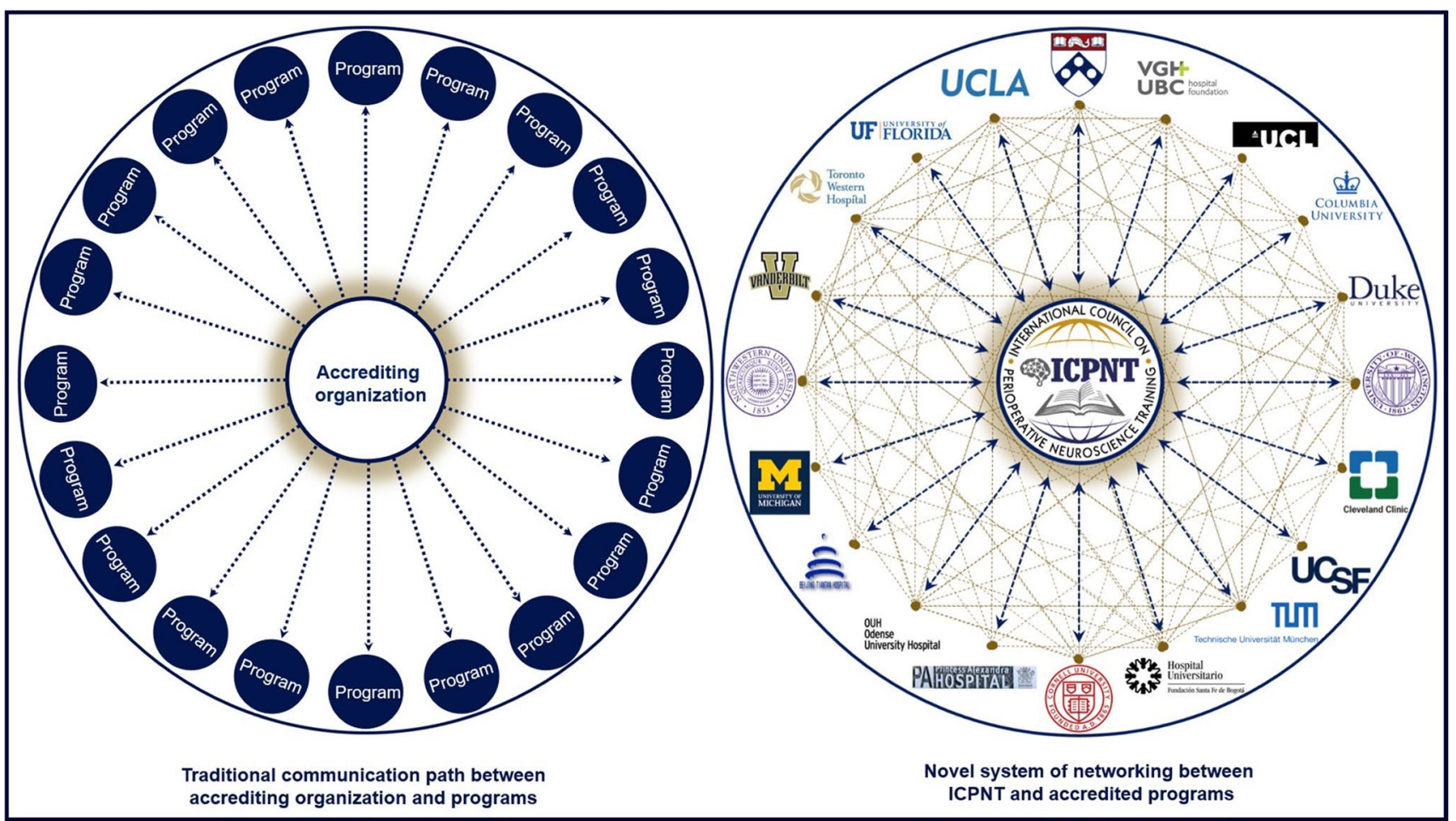

Fig. 1 Facilitation of an interprogram network by the ICPNT. Traditional vs. ICPNT network-driven communication pathway between accrediting organization and accredited academic programs. Currently accredited institutions: Beijing Tiantan Hospital, Beijing, China; Cleveland clinic Foundation, Cleveland, USA; Columbia University Medical Center, New York, USA; Duke University, Durham, USA; Hospital Universitario Fundación Santafe de Bogotá, Bogota, Columbia; Northwestern University, Chicago, USA; Odense University, Denmark; Princess Alexandra Hospital, Queensland, Australia; Technical University Munich School of Medicine, Munich, Germany;

Pillar 1-The care of neurosurgical patients: This involves the disciplines of neuroanesthesia, neurocritical care, and neuromonitoring of patients undergoing neurosurgical procedures and interventional neuroradiologic procedures.

Pillar 2-The foundational neuroscience of anesthesiology: The target of all anesthesia is the brain and any study and research of peripheral nerves, the spinal cord, subcortical arousal systems, thalamocortical and corticocortical networks supporting consciousness, pain networks, memory systems in the medial temporal lobe, the neuromuscular junction, and the autonomic nervous system would all come under the second pillar.

Pillar 3-Neurologic outcomes of non-neurosurgical procedures: This includes taking care of the neurologically impaired patient undergoing non-neurologic surgery. The trained neuro-anesthesiologist should be an expert in questions related to anesthetic neurotoxicity, delirium, cognitive dysfunction, stroke and perioperative brain health. This above description of the three pillars
Toronto Western Hospital, University of Toronto, Toronto, Canada; University college of London, London, UK; Universitat de Barcelona Neuroanesthesiology, Barcelona, Spain; University of California Los Angeles, Los Angeles, USA; University of California San Francisco, San Francisco, USA; University of Colorado, Denver, USA; University of Florida, Gainesville, Florida; University of Michigan, Ann Arbor, USA; University of Pennsylvania, Pennsylvania, USA; University of Washington, Seattle, WA; Vancouver General Hospital, Vancouver, USA; Vanderbilt University, Nashville, USA; Weill Cornell Medical College, New York, USA.

of neuro-anesthesiology then becomes the foundation on which structured and standardized training can be built.

In a confidential survey [21] which was distributed to an international cohort of anesthesiology attendings and trainees it was observed that despite advances in the field of perioperative neuroscience, there was a low interest amongst anesthesiology trainees to pursue subspecialty training in neuro-anesthesiology. Perceived barriers to neuro-anesthesiology fellowship training were perceptions that residency provides adequate training in neuro-anesthesiology, a unique skill set, or additional knowledge is not acquired with the extra year of training, and that there are limited job opportunities available to those with neuroanesthesiology fellowship training. Respondents believe that increased opportunities for research and greater exposure to neurocritical care and neurological monitoring methods would increase interest in neuroanesthesiology fellowship training. These findings may be used to guide curricular development and standardize training in neuroanesthesiology. 
In the light of all this information. It may be very timely to structure education around the three pillars and also offer training in technical skills such as scalp blocks, erector spinae blocks, learning modalities of monitoring like processed EEG, trans cranial Doppler, cerebral oximetry etc. Education should also focus on development of enhanced recovery after surgery (ERAS) protocols and perioperative brain health in the aging population thus making the fellowship geared towards the content of perioperative neuroscience. Research in basic neuroscience should also be offered for better understanding of the fundamental aspects of the neurosciences.

\section{Development of the Neuroanesthesia Program Relations Committee}

One of the unique advantages of ICPNT is a collaborative sharing of educational, scientific and professional resources between programs. With this in mind, the Neuroanesthesia Program relations committee (NPR) was created. The NPR creates an international collaborative platform for the accredited programs which may vary widely in their neuroanesthesia practices, to share academic resources and to learn from each other's experiences. This program represents an opportunity for fellows and faculty to gain worldwide exposure of patient management strategies. We believe this is one of the first such innovative educational efforts in sub-specialty education. The mission of the NPR committee is to foster academic and professional relations and networking among ICPNT accredited programs. This is probably the first such global collaboration offered by an accreditation body in the subspecialties of anesthesiology.

The committee organizes monthly educational sessions for the program faculty and fellows (Table 1). Journal clubs, interesting case discussions, etc.are the features of these online webinars with the accredited programs participating, presenting and collaborating. Additionally, workshops on peer review of journal articles and evaluation and feedback of fellows using milestones are conducted online for the benefit and education of the program directors. This creates a common virtual platform or stage which supports the growth of education in neuroanesthesia. Every effort is being made to conduct these webinars at a time which is convenient internationally considering that each accredited program is in a different time zone. The committee also organizes hands on workshops and sessions during the SNACC annual meeting.

During the covid pandemic, the committee reached out to fellowship programs around the world to survey the impact of the covid-19 pandemic on clinical training, education and the emotional wellness of the trainees in neuroanesthesia. The results published documented history as it happened and would also serve as evidence to show how programs used innovative ways to handle the crises at its peak [22].

The future vision of the NPR committee is to help programs collaborate with each other on research creating opportunities for fellows to participate in multicentric studies and offer extramural opportunities to obtain additional training. An example would be a program offering training in transcranial Doppler monitoring to another program which may not have this freely available to their trainees.

\section{Curriculum and Core Competencies of Neuroanesthesia Training Making Standardization a Practical Reality}

Similar to many other subspecialties in anesthesiology [23-25], the aim of a standardized ICPNT fellowship training curriculum is to develop an international cohort of experts and leaders with the required clinical, research, and educational foundation in perioperative neuroscience to improve patient outcome and health care delivery.

According to the data obtained from the 2010 survey to all SNACC members, the top choices for the curriculum included core training in clinical neuroanesthesia, career development, neurocritical care, and intraoperative neuromonitoring [4]. Soon after the survey, the first formal guidelines for the neuroanesthesiology fellowship training took shape by the SNACC task force in 2013 [10]. To explore the potential of accreditation of neuroanesthesiology fellowship through the American College of Graduate Medical Education (ACGME), the task force developed a 1-year curriculum based on ACGME competencies to lay the foundation. The curriculum consisted of essential elements voted by the SNACC members in 2010. It was based on the unit of 4-week modules, including six modules of clinical neuroanesthesia, and one module of each of the following areas: neurocritical care, neuroradiology, neuromonitoring, neuroscience scholarship. The remaining time was allowed for electives, such as transcranial Doppler ultrasonography, pediatric neuroanesthesiology, and additional training in neurocritical care, general anesthesiology, or scholarship. These guidelines represented the first step in standardization of basic requirements in fellowship training.

Similar enthusiasm has been reported by the neuroanesthesia communities around the world, regarding standardization of the curriculum for the fellowship training. The consensus from a Europe-wide-survey showed two-thirds of respondents agreeing that neuro-oncology surgery, skullbase neurosurgery, spinal surgery, awake craniotomies, sitting position surgery, interventional neuroradiology, epilepsy surgery, functional neurosurgery and neurocritical care should be mandatory in the fellowship program [14]. The results from the specialty-wide survey in Canada reported 
the majority support for the standardization of the fellowship goals and objectives, although it was not in favor of national accreditation given the limit in number of centers, funding and Canadian resident candidates in the country [13].

The ICPNT is committed to develop a SNACC-based, international neuroanesthesiology fellowship accreditation service [1]. Because of its international and non-ACGME related nature, ICPNT has drawn widespread interest across continents in the training of perioperative Neuroscience. As such the ICPNT is pioneering the notion that an internationally accepted standard for neuroanesthesia fellowships can be created. A writing committee has created the foundational documents for this organization based on past publications of SNACC members $[4,10]$. The program requirements are written to establish a minimum requirement in curriculum of clinical experiences and scholarly activities to comprise an accredited neuroanesthesiology fellowship, while keeping the goal to embrace international differences in regional training culture and regulations, and to allow for different methods of funding mechanisms. https://icpnt.net/ At the early development stage, four rounds of pilot program reviews and accreditation have helped ICPNT to strengthen the program requirements, governing procedures, and applications and review process, despite regional variations in different parts of the world.

The ICPNT curriculum (Table 2) entails that the fellow must be cognitively and technically competent, in all areas of patient care that requires the special expertise of the neuroanesthesiologist: pre-anesthetic evaluation, intraoperative management and postoperative care of patients undergoing neurosurgical, interventional neuroradiology procedures, spine and Intraoperative Neuromonitoring. It specifies the minimum experience of general and complex cases in each of the neurosurgical subcategories, including tumor, neurovascular, functional, spine, and special neurosurgical positions, for example, craniotomy in sitting position. The fellow

Table 2 Summary of the curricular guidelines endorsed by the ICPNT( www.ICPNT.NET). (Created by the ICPNT guidelines writing committee)

Curricular and Cognitive Competencies in Neuroanesthesia

Perioperative anesthetic care Assessment

Intraoperative management unique to Neuroanesthesia

Intraoperative management in Spine surgery

Postoperative management

Clinical procedures( interpretation and consultation of intraoperative neuromonitoring, precordial doppler, transcranial doppler, scalp blocks, cerebral oximetry etc

Neurocritical care module

Evaluate and manage critical neurological illnesses

Manage spinal and ventriculostomy drains, as well as intracranial pressure (ICP) monitors

Evaluate and treat perioperative morbidities in neurological injury patients

Safely transport and monitor critically ill neurological patients

Evaluate and manage postoperative pain and implement analgesic regimens tailored to the limitations imposed by the patient's neurological status

Interventional Neuroradiology Anesthesia management- Understand and provide appropriate anesthetic care for various interventional neuroradiology procedures; understand anticoagulation and reversal

Endovascular Procedure Module- Assess MRI and apply knowledge to the interventional procedure to be performed; Discuss the procedure with risks and benefits with the patient or the designated medical power of attorney; Assist the neurointerventionalist as directed iv. Understand the decisions and outcomes from the intervention; Follow and assist in management of postoperative care; Be able to identify and begin care intervention for common intravascular postoperative complications

Cognitive Module

Neuroanatomy, Neurophysiology, Neuropathology

Neuropharmacology

Neurologic injury Classification and Grading

Applied Medical Knowledge

System Learning, Business Management, and Ethics (Optional)

Educational Activities

Didactic conferences

Quality Assurance conferences

Skills/Simulation conferences

Outside conferences

Research

Educational methods

Problem Based Learning Discussion

Daily or weekly clinical case Presentations

Informal Case presentation of Daily clinical activity to residents

Self-learning conferences

Formal lectures

Formal journal club 
should also demonstrate neurocritical care skill, and should be competent to provide consultation, specialized recommendation or services for neurologically complex patients undergoing non-neurosurgical procedures. The ICPNT goal is to prepare fellows to specialize in perioperative care of neurosurgical interventions, be able to independently manage complex and rare neurosurgical cases, as well as neurological crisis, at both cognitive and skill levels.

In addition to the acquisition of clinical and scholarly expertise, another important aspect of the ICPNT curriculum is to stage the fellow supervision levels from direct, indirect, and to oversight. The progression of supervisory training will prepare the fellows to adopt to junior faculty roles later in the training. The program encourages fellows to gain skills in teaching, supervising, and professionalism, in preparation to become future generations of educators and leaders in perioperative neuroscience.

\section{Assessment and Evaluation Using Neuroanesthesia Milestones-an Essential Component of Standardization as the Future of the Subspecialty Evolves}

Competency is the term often used to refer to a specific area of performance that can be described and measured, such as the competencies that were identified by the Outcomes Project of the ACGME: patient care, medical knowledge, professionalism, practice-based learning and improvement, systems-based practice, and interpersonal and communication skills [25]. A Milestone is a significant point in development. Milestone refers to a point along a continuum of a competency or sub competency; milestones are clearly described and are usually specialty- specific. [25].

For accreditation purposes, the Milestones are competency-based developmental outcomes (e.g., knowledge, skills, attitudes, and performance) that can be demonstrated progressively by residents/fellows from the beginning of their education through graduation to the unsupervised practice of their specialties. As the conceptual framework of the milestones describe they are designed to assist residency programs, residents/fellows and accreditation organizations with a progression of competency from entry-level to exceptional skill [26].

In 2009, the ACGME introduced the concept of competency-based assessments, or milestones, as part of the Next Accreditation System in partnership with the member boards of the American Board of Medical Specialties [24]. A milestone is a significant point in development. The Milestones provide residency programs with a rich descriptive, developmental framework for 1) Clinical Competency Committees (CCCs), guide curriculum development of the residency or fellowship, support better assessment practices and enhance opportunities for early identification of struggling residents/ fellows. 2) For residents/fellows, the Milestones provide more explicit expectations for performance, support better self-directed assessment and learning and facilitate better feedback for professional development and 3) for accreditation, the Milestones allow for continuous monitoring of programs and lengthening of site visit cycles, enhance public accountability-report at a national level on aggregate competency outcomes by specialty [26].

In 2014, 25 specific anesthesiology milestones were released. These milestones are use across all the residency programs, but they are not sub-specialty specific. This led to several members of the SNACC expressing the need for Neuroanesthesiology-specific milestones for resident education. As a result, the SNACC commissioned a task force to adapt the ACGME anesthesiology milestones for use in Neuroanesthesiology to facilitate and improve its use during the subspecialty residency training in 2019 [27]. A 7-member task force and an 8-member advisory committee comprising by senior Neuroanesthesiology educators in the US was appointed. The task force developed the Neuroanesthesiology milestones based on the 25 existing ACGME anesthesia milestones. The task force reviewed all the feedback obtained from the SNACC membership and finally recommended 12 Neuroanesthesiology specific milestones in 5 major ACGME domains that were identified as most pertinent to the subspecialty resident rotation. These pertain to patient care ( 7 milestones), medical knowledge (2 milestones), practice-based learning and improvement (1 milestone), and interpersonal and communication skills (2 milestones). The major strength of the SNACC milestones is the rigorous process of their development. This included piloting by a large number of faculty and residents in Anesthesiology departments representing the diversity of training programs in the US, as well as inputs from the SNACC membership representing content expertise [28]. A major limitation of the SNACC milestones is that they are based on the competency model used in the USA and was piloted only in USA. Other methods of competence-based assessment are used in other countries. There are also other evaluation methods being used across various programs. The opportunity here is of creating a universal evaluation tool by collaboration.

\section{Conclusion}

Given the absence of certification and accreditation data, we do not have solid data on the number of and need for properly trained neuroanesthesiologists. The ASA(American society of Anesthesiology) worked with the Anesthesia Quality institute to overview the practice of neuroanesthesia in the US publishing a summary in the 
Journal of Neurosurgical anesthesiology. Unpublished collected data indicated an overall growth in procedures from 2010 to 2014 from 2.15 to 3.68 million procedures [29]. This included significant increase in brain tumor surgery, carotid endarterectomy and interventional radiology procedures. There are also indirect signs of increased interest and need for neuroanesthesiologists based on the successful initiation and continuation of subspecialty journals (Journal of Neurosurgical Anesthesiology and the Indian journal of Neuroanesthesia and Critical Care), several successful textbooks and manuals of Neuroanesthesiology, the large number of neuroanesthesia societies world-wide and increasing membership in SNACC.

ICPNT has now created the opportunity and path to global collaboration which would lead to standardization of neuroanesthesia training and education. This could mark the beginning of a new era for neuroanesthesiology. The need of the hour is to create an urgent awareness of the specialty of neuroanesthesia, emphasis on standardization of education through a central body (ICPNT) which is international in scope, embracing the concept of the three pillars of neuroanesthesia and collaborative communication of educational experiences, assessment and evaluation of trainees in a standardized manner. While there is a need to increase the number of fellowship trained neuroanesthesiologists through a standardized system, the drive needs to come from departments of anesthesiology seeking to do complex neurological cases and the demand from our neurosurgical colleagues.

More information, including an FAQ list, is available at the ICPNT website, www.icpnt.net as well as the ICPNT twitter feed @ icpnt. We welcome advice from the international academic neuroanesthesiology community.

Acknowledgements The ICPNT writing committee: W Andrew Kofke, Chair, Lara Ferrario, Secretary-Treasurer, Rafi Avitsian, John Patrick Bebawy, Maria Bustillo, Alana Flexman, Leslie Jameson, Phuping A Kavipat, Chanhung Lee, Nan Lin, Coleen Moran, Mike Nathanson, Chanannait Paisansathan, Jeffrey Pasternak, Shobana Rajan, Shilpa Rao, Steven Robischek, Deepak Sharma, Ricard Valero, Stewart Hinckley (Ruggles Advisor)

Figure 1 collaborative framework of accredited programs Figure contributed by Jovany Cruz Navarro, MD Assistant Professor Department of Anesthesiology Department of Neurosurgery Baylor College of Medicine Houston TX, USA. Figures and tables are originals-belong to ICPNT — no need for permission

\section{Declarations}

Human and Animal Rights and Informed Consent This article does not contain any studies with human or animal subjects performed by any of the authors.

Conflict of Interest The authors declare no competing interests.

\section{References}

Papers of particular interest, published recently, have been highlighted as:

• Of major importance

1.•• Ferrario L, Kofke WA. Standardized Accreditation of Neuroanesthesiology Fellowship Programs Worldwide : The International Council on Perioperative Neuroscience Training. Neurosurg Anesthesiol. 2019;31(3):267-9. https://doi.org/10.1097/ANA.00000 00000000605. An important article which discusses that lack of standardization of education would generate a major disparity in the degree of expertise acquired among experts in a particular field.

2. Fellowships. Society of Neuroscience in Anesthesiology and critical care. https://www.snacc.org/fellows-and-residents/fellowships/ Accessed Apr 22, 2021.

3. Khandelwal A, Jangra K, Katikar MD, Durga P, Rao GSUM. Choosing Neuroanaesthesia as a career: Marching towards new horizons. Indian J Anaesth. 2021;65(1):35-42. https://doi.org/10. 4103/ija.IJA_1531_20.

4. Mashour GA, Lauer K, Greenfield MLVH, Vavilala M, Avitsian R, Kofke A, ... Brambrink A. Accreditation and standardization of neuroanesthesia fellowship programs: Results of a specialty-wide survey. J Neurosurg Anesthesiol. 2010;22(3):252-255. https://doi. org/10.1097/ANA.0b013e3181d908d7.

5. Albin MS. Celebrating silver: the genesis of a neuroanesthesiology society. NAS-->SNANSC-->SNACC. Neuroanesthesia Society. Society of Neurosurgical Anesthesia and Neurological Supportive Care. Society of Neurosurgical Anesthesia and Critical Care. J Neurosurg Anesthesiol. 1997;9(4):296-307.

6. Bates J, Schrewe B, Ellaway RH, Teunissen PW, Watling C. Embracing standardisation and contextualisation in medical education. Med Educ. 2019;53(1):15-24.

7. Kofke WA. Celebrating ruby: 40 years of $\mathrm{NAS} \rightarrow$ SNANSC $\rightarrow$ SNACC $\rightarrow$ SNACC. J Neurosurg Anesthesiol. 2012;24(4):260-80. https://doi.org/10.1097/ANA.0b013e3182 $68005 \mathrm{~b}$.

8. Albin MS. Mission (not yet) Accomplished! SNACC Newsletter. 2008;36:1-3.

9. Lanier WL. The history of neuroanesthesiology: the people, pursuits, and practices. J Neurosurg Anesthesiol. 2012;24(4):281-99. https://doi.org/10.1097/ANA.0b013e3182697743.

10. Mashour GA, Avitsian R, Lauer KK, Soriano SG, Sharma D, Koht A, Crosby G. Neuroanesthesiology fellowship training: Curricular guidelines from the society for neuroscience in anesthesiology and critical care. J Neurosurg Anesthesiol. 2013;25(1):1-7. https://doi. org/10.1097/ANA.0b013e3182785d5d.

11. Fàbregas N, Salvador L, Valero R, Carrero E, Gomar C, Nalda M. Analysis of the current status of neuroanesthesia in Spain using a national survey. Rev Esp Anestesiol Reanim. 1998;45:166-71.

12. Nathanson M. Working together to improve patient care: Guidelines for neuroanaesthesia. Rev Esp Anestesiol Reanim. 2012;59:1-2. https://doi.org/10.1016/S0034-9356(12)70001-4.

13. Sewell D, Henderson C, Tremblay MH, Pellerin H, Flexman AM. Survey of neuroanesthesia fellowships in Canada. Can J Anesth. 2017;64(3):323-4. https://doi.org/10.1007/s12630-016-0751-6.

14. Valero R, Nathanson MH, Willner D, Fàbregas N, Bilotta F, for the Neuroscience Subcommittee of the European Society of Anaesthesiology, t. E. N., \& Group, C. C. I. Developing standardised advanced training in neuroanaesthesia: Results of a Europewide survey. Eur J Anaesthesiol (EJA). 2017;34(2):51-3. https:// doi.org/10.1097/eja.0000000000000530. 
15. Niño de Mejía MC. Neuroanestesia, libros en línea. Retrieved from https://www.sbahq.org/resources/pdf/arquivos/ebook/neuro anestesia-libro-en-linea.pdf. 2015.

16. González Villavelázquez ML, DLLIGL. 40 Years of Neuroanesthesiology in Mexico: Notes for History. Anesth Mexico. 2018;30(3):1-5.

17. Raksakietisak MW, Pichaya, Akavipat, Phuping. A Letter from Thai Society for Neuroanesthesia (TSNA) Fall 2018. Retrieved from https://www.snacc.org/wp-content/uploads/2018/fall/artic le5.html.

18. Saw AS. Neuroanaesthesia Services of Myanmar: The Current Status from a Humble Beginning. SNACC Newsletter. 2018. Retrieved from https://www.snacc.org/wp-content/uploads/2018/ fall/article6.html.

19. Mashour GA. The Three Pillars of Neuroanesthesiology. ASA Monitor. 2017;81:48.

20. Mashour GA. The Three Pillars of Neuroanesthesiology. SNACC Winter newsletter 2016-PUB-1.pdf.

21. Rajan S, Theard MA, Easdown J, Goyal K, Pasternak JJ. Perceived Benefits and Barriers to a Career in Neuroanesthesiology: A Pilot Survey of Anesthesiology Clinicians. J Neurosurg Anesthesiol. 2019.

22. Rajan S, Bebawy J, Avitsian R, Lee CZ, Rath G, Luoma A, Bilotta F, Pierce JT, Kofke WA .The Impact of the Global SARS-CoV-2 (COVID-19) Pandemic on Neuroanesthesiology Fellowship Programs Worldwide and the Potential Future Role for ICPNT Accreditation.

23. Capdeville M, Ural KG, et al. The educational evolution of fellowship training in cardiothoracic anesthesiology - perspectives from program directors around the United States. J Cardiothorac Vasc Anesth. 2018;32:607-20.
24. Mariano ER. The road to accreditation for fellowship training in regional anesthesiology and acute pain medicine. Curr Opin Anaesthesiol. 2018;31(5):643-8.

25. Nguyen-Buckley C, et al. Recommendations From the Society for the Advancement of Transplant Anesthesiology: Liver Transplant Anesthesiology Fellowship Core Competencies and Milestones. Semin Cardiothorac Vasc Anesth. 2019;23(4):399-408.

26. Sklar DP. Competencies, milestones, and entrustable professional activities: what they are, what they could be. Acad Med. 2015;90(4):395-7.

27. Ambardekar ALT, Brennan K, McKenzie-Brown AM, et al. Anesthesiology Milestones. The Accreditation Council for Graduate Medical Education. 2020 [cited 2020 November 2020] Second Revision.

28.• Sharma D, et al. Society for Neuroscience in Anesthesiology \& Critical Care (SNACC) Neuroanesthesiology Education Milestones for Resident Education. J Neurosurg Anesthesiol. 2019;31(3):33741. The SNACC Neuroanesthesiology milestones provide a framework for reviewing resident performance during Neuroanesthesiology subspecialty training.

29. Farag E, Westlake B, Dutton RP, Mascha EJ, Kofke WA. The ASA Committee for Neuroanesthesia and Anesthesia Quality Institute: Report for Demographic Patterns for Neurosurgical Anesthesia Practice in the United States. J Neurosurg Anesthesiol. 2018;30(2):189-91. https://doi.org/10.1097/ANA.00000 00000000437.

Publisher's Note Springer Nature remains neutral with regard to jurisdictional claims in published maps and institutional affiliations.

\section{Authors and Affiliations}

\section{Shobana Rajan ${ }^{1} \cdot$ Maria Bustillo $^{2} \cdot$ Chanhung Lee $^{3} \cdot$ W Andrew Kofke $^{4}$}

Maria Bustillo

bustilo@med.cornell.edu

Chanhung Lee

Chanhung.Lee@ucsf.edu

W Andrew Kofke

kofkea@pennmedicine.upenn.edu

1 Department of Anesthesiology, University of Texas Health Science center at Hosuton, 6431 Fannin Street, Houston, Texas, USA
2 Department of Anesthesiology, Weill Cornell Medicine, 525 East 68th St, P-309, New York, NY 10065, USA

3 UCSF Department of Anesthesia \& Perioperative Care, 521 Parnassus, Floor 04, Box 0648, San Francisco, CA 94143 , USA

4 Department of Anesthesiology, University of Pennsylvania, 3400 spruce st, Philadelphia, PA 19104, USA 